\title{
Study on the clinical value of miRNA98b as a potential biomarker for osteoarthritis.
}

\author{
Qian Wang ${ }^{1}$, Xiaoqing $\mathrm{Ma}^{2}$, Zhongyang $\mathrm{Xu}^{1}$, Weidong Zheng ${ }^{\text {* }}$ \\ ${ }^{1}$ Department of Spinal Surgery, the First People's Hospital of Jining, Jining, Shandong, PR China \\ ${ }^{2}$ Department of Endocrine, the First People's Hospital of Jining, Jining, Shandong, PR China \\ ${ }^{3}$ Department of Traumatic Surgery, the First People's Hospital of Jining, Jining, Shandong, PR China
}

\begin{abstract}
Objective: Osteoarthritis is a serious threat to the health of patients. Biomarkers have important significance in the diagnosis of bone arthritis. Bone mineral density is one of the important indicators for the detection of bone joint inflammation, but its detection is time-consuming and laborious. MicroRNA regulates cancer and inflammation. This study was to investigate the potential value of biomarkers miRNA98b in the diagnosis of early osteoarthritis.

Methods: 69 cases of patients with osteoarthritis and 69 cases of healthy volunteers were regarded as research objects. Etidronate disodium had been continuously administrated for $4 \mathrm{w}$. The patient's bone density reached to the normal value which was regarded as effectiveness of the treatment. The miRNA98b level before and after therapy in blood cells of patients with osteoarthritis and healthy volunteers was detected by real time RT-PCR. The correlation between miRNA98b levels and osteoarthritis was analysed.

Results: mRNA level of miRNA98b in blood cells of patients with osteoarthritis was higher than that of healthy volunteers. After being continuously administrated for $2 \mathrm{w}$, mRNA of miRNA98b in blood cells decreased, there was no significant change in the level of miRNA98b in blood cells of healthy volunteers. The level of miRNA98b in the blood cells of patients with severe osteoarthritis is higher than that in the blood cells of patients with general osteoarthritis, the difference was statistically significant $(P<0.05)$. The level of miRNA98b in the blood cells of patients with osteoarthritis has a positive correlation with the severity of osteoarthritis.

Conclusion: MiRNA98b in blood cells of patients with osteoarthritis may be a potential biomarker for osteoarthritis. The level of miRNA98b was correlated with the severity of osteoarthritis.
\end{abstract}

Keywords: Osteoarthritis, miRNA98b, Diagnosis.

Accepted on November 10, 2017

\section{Introduction}

Osteoarthritis is a degenerative disease, which is caused by various factors such as age, obesity, strain, trauma, congenital deformity and so on [1,2]. Osteoarthritis brings economic burden and pain to the patient [3]. Another symptom is the stiffness of the joint, which often maintain a certain position in the morning to get up or a day after a long time. There are redness and swelling of joints, pain, activity with a sense of friction or "point" sound in osteoarthritis, severe cases can lead to muscle atrophy and joint deformities.

Early detection and therapy of osteoarthritis is the best choice. When it is necessary to check the diagnosis of synovial fluid, $\mathrm{X}$-ray changes could not explain the primary osteoarthritis. According to the development of OA, diagnosis and classification were carried out [4]. Therefore, the early diagnosis and treatment of osteoarthritis has important clinical significance. Research suggested that Bone Mineral Content (BMC) or Bone Mineral Density (BMD) has diagnosed value to osteoarthritis $[5,6]$. BMD or BMC is the diagnostic criteria for osteoarthritis, which test sites in patients with axial bone or peripheral bone [7]. However, this method is tedious, timeconsuming and laborious. The clinical need for more effective detection of osteoarthritis indicators and methods [8].

Biomarker [1] explore the importance of pathogenesis [9]. By measuring the level of type I or urine pyridine (pyridine PYD) $\mathrm{C}$ and N CTX-I can reflect the status of its metabolism. Serum calcitonin is a kind of important immune protein in serum, and it is often used as a marker of osteoarthritis. The existing bone metabolism markers were used to reflect the situation of osteoarthritis. More and more scholars are devoted to explore the specificity and sensitivity of the metabolism of cartilage and synovial cells. Collagen type II collagen synthesis metabolism can be reflected by the detection of multiple 
markers, which is the most important part of the carboxyl terminal peptide (CTXII). A large number of studies have demonstrated that the levels of OA II in RA patients were significantly higher in the urine and CTX. It was found that OA increased the early type II collagen synthesis by measuring serum type III collagen (II type c-terminalpropeptide PIICP, type II PRO COLLAGEN carboxyl terminal peptide). Researchers [2] detected PIIANP by ELISA, it was found that the level of PIIANP obviously decreased in OA patients. Deberg [3] found that two kinds of degradation products of collagen type II collagen peptides: CTX II and PIIANP. Charni [4] found that the level of PIIANP in patients was significantly higher than that in healthy control group (Table 1). Double foot type of metabolic markers of bone, cartilage and synovial membrane.

Bone density is one of the indexes of osteoarthritis, but its measurement is time-consuming and laborious. The biomarkers of osteoarthritis are I type urinary pyridine (pyridine PYD), the $\mathrm{C}$ and $\mathrm{N}$ end peptide collagen cross linking peptide (CTX-I) is an indicator of the degree of the osteoarthritis, but detection of PYD and the end need ELISA kit and microplate reader, which price is higher and is not suitable for economic conditions a little worse [10,11]. As a marker of osteoarthritis, serum calcitonin (calcitonin) does not apply to all groups of people, and it is not applicable to some patients. Clinical need for high sensitivity, specificity, low cost of osteoarthritis biomarkers.

MiRNA is a small molecule that is widely present in animals, plants, microorganisms, and other species, previous studies suggest that miRNA has no function, however, with the continuous deepening of research, people gradually found that it has a wide range of biological functions, such as cell cycle, cell growth, apoptosis, necrosis, cell toxicity, cell proliferation, cell autophagy and so on. The latest research shows that miRNA has a close relationship with a variety of physiological and pathological conditions. The present study suggests that miRNA is associated with the occurrence, development and prognosis of cancer, which can be used as a molecular marker of cancer $[12,13]$. For example, microrna-18a is a molecular marker of prostate cancer [5]; microRNA 25, microRNA 145 and microRNA 210 are molecular markers of liver cancer. MicroRNA-200c-141 is a molecular marker of lung cancer [6]. It suggests that microRNA may be a marker for patients with osteoarthritis. It has known miRNA98b is related to the inflammatory state of the cell and the body [14]. But the occurrence of osteoarthritis is closely related to the inflammatory reaction of the body [15]. Therefore, this study discusses the potential value of biomarkers miRNA98b in the diagnosis of early osteoarthritis.

\section{Materials and Methods}

\section{Experiment object}

We treated 69 cases of osteoarthritis patients as experimental group, who were received and cured in orthopaedics in the First People's Hospital of Jining from June 2014 to June 2016.
Blood in patients with osteoarthritis before and after treatment were collected. At the same time, 69 healthy volunteers were served as the control group. Etidronate disodium had been continuously taken for $2 \mathrm{w}$. Patients with bone mineral density reached normal values to judge the effectiveness of treatment. The inclusion and exclusion criteria for osteoarthritis were graded and diagnosed according to the recommendations of the WHO in 2015.

This study has been pre-approved by the ethical committee of the First People's Hospital of Jining. All subjects have signed the consent forms before recruitment in this study.

\section{Preparation of blood samples of patients with osteoarthritis}

Venous blood $15 \mathrm{ml}$ was extracted in the early morning for all patients with an empty stomach. Blood cells were collected and stored in $-20^{\circ} \mathrm{C}$ set aside.

\section{RT-PCR}

The total RNA of blood cells was extracted by using Trizol reagent accroding conventional method, RT-PCR was carried out according to the instruction of reverse transcription kit (Beijing Tiangen biotech company) [16]. The definite means were as follows: the venous blood of patients with osteoarthritis was firstly collected, $600 \mathrm{rpm}$ centrifugation for 5 minutes, and the supernatant was collected and the total RNA was extracted. Reverse transcription and PCR were carried out according to general process. Primers of MiRNA98b and internal reference actin were synthesized by Beijing three Bo Polygala biotechnology company, the sequences were as follows respectively: 5'-GCTGCGCTACAATGATGTGG-3' and 5'-GGCTGGAAAGGAAAGAGTGC-3’, 5‘CGTGGAGTCGGCAACTCAACTGGTGTTTCAGTTGAG-3 , and 5'. GTGGAGTCGGCAATTCCTCAACTGGTGTCAGTTGAG AGGGATTC-3'.

\section{Agarose gel assay for RT-PCR products}

PCR products were detected by electrophoresis with $80 \mathrm{v}$, which were analysed in gel electrophoresis gel imaging system (BD Instruments Inc.). The results of electrophoresis were analysed into statistic (imagej $1.48 \mu$, National Institutes of health, U.S.A), gray value of RT-PCR product was analysed, the ratio of the gray value and internal control was regarded as relative expression.

\section{Statistical analysis}

At first, the numerical variables of the experiment were tested, data consistent with the normal distribution was expressed as mean plus or minus standard error representation, $\mathrm{P}<0.05$ represented a significant difference. 


\section{Results}

\section{Basic information of patients with osteoarthritis}

A total of 156 patients with osteoarthritis and 156 healthy volunteers were enrolled in this study (Table 1).

\section{RT-PCR test results of miRNA98b expression in blood cells of patients with osteoarthritis}

As shown in Figure 1, the RT-PCR test results were shown, the mRNA expression level of miRNA98b in the blood cells of patients with osteoarthritis was significantly higher than that in healthy volunteers, and the difference was significant between the two groups.

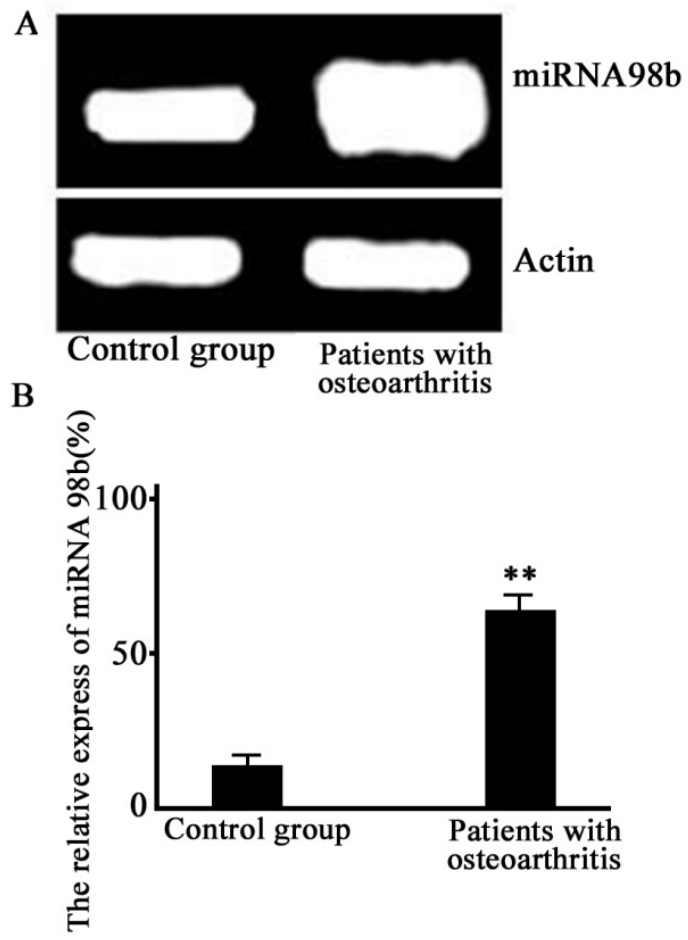

Figure 1. RT-PCR test results of miRNA98b expression in blood cells of patients with osteoarthritis. Note: ${ }^{* *} P<0.01$ : Compared with control group, the difference was significant.

\section{MRNA level of miRNA98b in blood cells of patients with osteoarthritis after treatment decreased}

As shown in Figure 2, after etidronate disodium had been continuously administrated for $2 \mathrm{w}$, mRNA levels of miRNA98b in the blood cells of patients with osteoarthritis were significantly decreased.

The level of miRNA98b in the blood cells of osteoarthritis patients was significantly positively correlated with the severity of osteoarthritis.

As shown in Figure 3, correlation analysis showed that the expression level of miRNA98b in the blood cells of patients with osteoarthritis was significantly positively correlated with the severity of osteoarthritis. Pearson correlation analysis results were shown in Figure 3, the correlation coefficient was 0.93 .

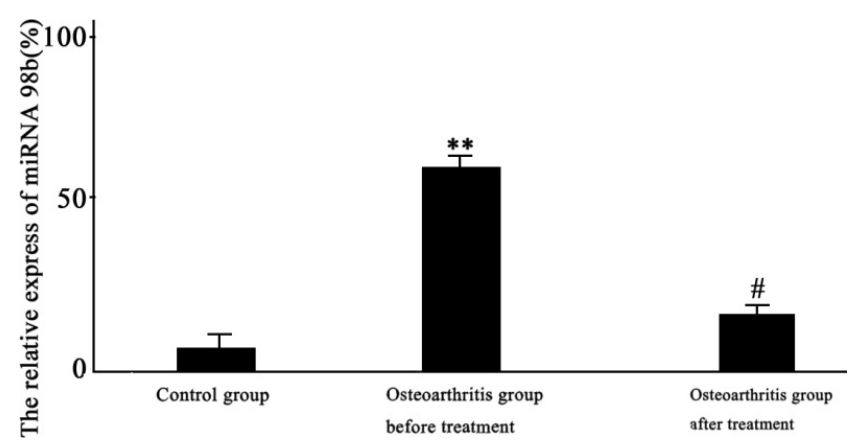

Figure 2. After etidronate disodium had been continuously administrated for $2 w, m R N A$ levels of miRNA98b in the blood cells of patients with osteoarthritis were significantly decreased. Note: ${ }^{* *} P<0.01$ : Compared with control group, the difference was significant. ${ }^{\#} P<0.05$ : Compared with osteoarthritis group before treatment, the difference was significant.

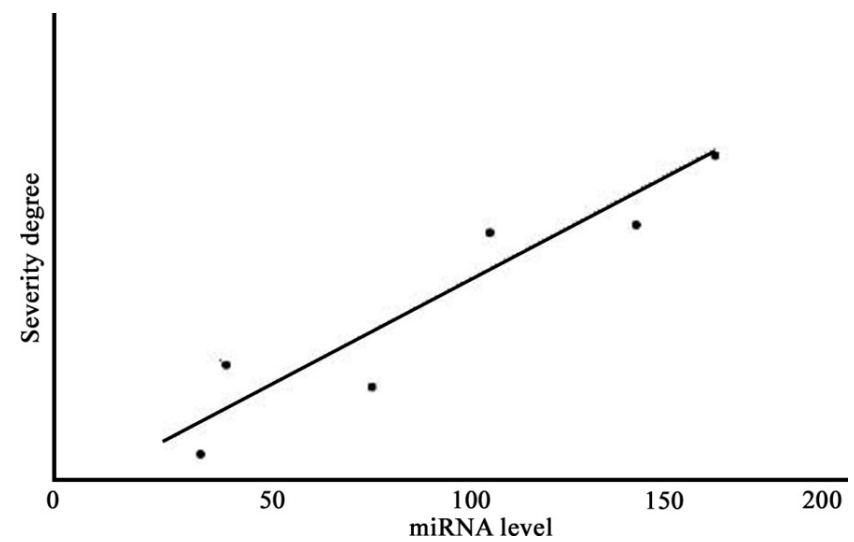

Figure 3. The level of miRNA98b in the blood cells of osteoarthritis patients was significantly positively correlated with the severity of osteoarthritis.

Table 1. Patients results $(\bar{x} \pm s)$.

\begin{tabular}{llll}
\hline Variable & $\begin{array}{l}\text { Osteoarthritis } \\
\text { group }\end{array}$ & Healthy volunteers group & $\mathbf{P}$ \\
\hline Male/female & $156 / 156$ & $156 / 156$ & 0.87 \\
\hline Ages (y) & $52.2 \pm 18.2$ & $56.2 \pm 17.3$ & 65 \\
\hline Average BMD & $608\left(\mathrm{~g} / \mathrm{cm}^{2}\right) \pm 0.3$ & $1219\left(\mathrm{~g} / \mathrm{cm}^{2}\right) \pm 1.7$ & 0.0018 \\
\hline $\begin{array}{l}\text { BMD after } \\
\text { therapy }\end{array}$ & $1005\left(\mathrm{~g} / \mathrm{cm}^{2}\right) \pm 5.5$ & $1309\left(\mathrm{~g} / \mathrm{cm}^{2}\right) \pm 4.6$ & 0.68 \\
\hline
\end{tabular}

\section{Discussion}

The early diagnosis and treatment of osteoarthritis is extremely important [17]. Because there is a lack of specificity and sensitivity of the biological markers of osteoarthritis [18], therefore, this study explores the possibility of miRNA98b as a biomarker for early diagnosis and prognosis of osteoarthritis.

Although most of the current studies of marker levels (CTXII, COMP, PYD, etc.) in the majority of OA individuals significantly increased, but in a certain proportion of patients 
have shown a normal level. This suggests that it was not sensitive enough of these markers as a diagnostic tool for hospital clinical automation. In patients without hip OA imaging characteristics of performance, COMP increasing in blood has relationship with some symptoms such as pain and stiff, but it was not found in patients with knee joints osteoarthritis [8]. COMP and NTX-I were found to be related to imaging progression in elderly women with OA, which level increased in hip OA women patients $4 \mathrm{y}$ after menopause [19]. The study found that the patient's knee joint trauma is closely related to osteoarthritis, microscopic observation showed that there was an early degeneration of cartilage in the patients with osteoarthritis, and the expression of PIICP was enhanced [20].

The severity of the disease is a marker for assessing the degree or extent of OA lesions, especially at a certain time point [3]. Serum protein expression was increased in OA patients [21-23]. In a large variety of ethnic groups, the study found that the levels of hyaluronic acid were significantly higher in males than that of females [24]. Another study, which included 291 white patients with OA of the knee, it was found a significant increase in blood COMP levels in patients with K-L and consistent with the patient's score [18]. In 324 postmenopausal women, the study showed that the level of II CTX in different parts of OA was different, which suggested that OA can be identified in different parts of the patient [25]. Deficiencies and shortcomings of this study are as follows: firstly, the sample sizes were small, future studies should further expand the number of osteoarthritis patients to further study the possibility of miRNA98b as an early diagnosis of osteoarthritis. Second, because the clinical practice of the majority of osteoarthritis patients were receiving chemotherapy and other treatment, whether miRNA98b expression levels were affected by the treatment to be further explored. Thirdly, this paper was lack of a conclusion that miRNA98bwas regarded as a target of osteoarthritis animal model to further verify the conclusions of this paper.

In conclusion, the project suggests that miRNA98b may be a specific biomarker in patients with osteoarthritis in the blood cells of patients with osteoarthritis. The level of miRNA98b was closely related to the severity of osteoarthritis.

\section{References}

1. Terencio MC. Chondroprotective effects of the combination chondroitin sulfate-glucosamine in a model of osteoarthritis induced by anterior cruciate ligament transection in ovariectomised rats. Biomed Pharmacother 2016; 79: 120-128.

2. Loeser RF. Association of urinary metabolites with radiographic progression of knee osteoarthritis in overweight and obese adults: an exploratory study. Osteoarthritis Cartil 2016; 1063-4584: 01072-01074.

3. Zhong H. T2 map signal variation predicts symptomatic osteoarthritis progression: data from the Osteoarthritis Initiative. Skeletal Radiol 2016; 45: 909-913.
4. Henrotin Y. Soluble biomarkers development in osteoarthritis: from discovery to personalized medicine. Biomarkers 2015; 20: 540-546.

5. Rahmati M. Inflammatory mediators in osteoarthritis: A critical review of the state-of-the-art, current prospects, and future challenges. Bone 2016; 85: 81-90.

6. Ren G. Applying computation biology and big data to develop multiplex diagnostics for complex chronic diseases such as osteoarthritis. Biomarkers 2015; 20: 533-539.

7. Eckstein F. A 20 years of progress and future of quantitative magnetic resonance imaging (qMRI) of cartilage and articular tissues-A personal perspective. Semin Arthritis Rheum 2015; 0049-0172: 00283-00288.

8. Chung HJ. Anti-osteoporotic activity of harpagide by regulation of bone formation in osteoblast cell culture and ovariectomy-induced bone loss mouse models. J Ethnopharmacol 2016; 179: 66-75.

9. Jiao Q. Cartilage oligomeric matrix protein and hyaluronic acid are sensitive serum biomarkers for early cartilage lesions in the knee joint. Biomarkers 2016; 21: 146-151.

10. Han W. Signal intensity alteration in the infrapatellar fat pad at baseline for the prediction of knee symptoms and structure in older adults: a cohort study. Ann Rheum Dis 2015; 26: 208360.

11. Van Spil WE. Associations of markers of matrix metabolism, inflammation markers, and adipokines with superior cam deformity of the hip and their relation with future hip osteoarthritis. Osteoarthritis Cartil 2015; 23: 1897-1905.

12. Runhaar J. Fibulin-3 fragments are prognostic biomarkers of osteoarthritis incidence in overweight and obese women. Osteoarthritis Cartil 2016; 24: 672-678.

13. Lee EJ. Interleukin-33 acts as a transcriptional repressor and extracellular cytokine in fibroblast-like synoviocytes in patients with rheumatoid arthritis. Cytokine 2016; 77: 35-43.

14. Buttgereit F. Non-surgical management of knee osteoarthritis: where are we now and where do we need to go? RMD Open 2015; 1: 000027.

15. Hunter D. Longitudinal validation of periarticular bone area and 3D shape as biomarkers for knee OA progression? Data from the FNIH OA Biomarkers Consortium. Ann Rheum Dis 2015; 19: 207602.

16. Gungen GO, Ardic F, Findikoglu G, Rota S. Effect of mud compress therapy on cartilage destruction detected by CTX-II in patients with knee osteoarthritis. J Back Musculoskelet Rehabil 2015; 6.

17. Eckstein F. Brief report: cartilage thickness change as an imaging biomarker of knee osteoarthritis progression: data from the foundation for the national institutes of health osteoarthritis biomarkers consortium. Arthritis Rheumatol 2015; 67: 3184-3189.

18. Mickiewicz B. Metabolic analysis of knee synovial fluid as a potential diagnostic approach for osteoarthritis. J Orthop Res 2015; 33: 1631-1638. 
19. Bagi CM. Correlation between $\mu \mathrm{CT}$ imaging, histology and functional capacity of the osteoarthritic knee in the rat model of osteoarthritis. J Transl Med 2015; 3: 276.

20. Tsai PH. Abnormal perfusion in patellofemoral subchondral bone marrow in the rat anterior cruciate ligament transection model of post-traumatic osteoarthritis: a dynamic contrast-enhanced magnetic resonance imaging study. Osteoarthritis Cartil 2016; 24: 129-133.

21. Arden N. Can we identify patients with high risk of osteoarthritis progression who will respond to treatment? A focus on biomarkers and frailty. Drugs Aging 2015; 32: 525-535.

22. Liu HF. Pulsed electromagnetic fields for postmenopausal osteoporosis and concomitant lumbar osteoarthritis in southwest China using proximal femur bone mineral density as the primary endpoint: study protocol for a randomized controlled trial. Trials 2015; 16: 265.

23. Yu H. Effect of advanced oxidation protein products on articular cartilage and synovium in a rabbit osteoarthritis model. Orthop Surg 2015; 7: 161-167.
24. Garciadiego-Cazares D. Regulation of $\alpha 5$ and $\alpha \mathrm{V}$ integrin expression by GDF-5 and BMP-7 in chondrocyte differentiation and osteoarthritis. PLoS One 2015; 10: 0127166 .

25. Kraus VB. OARSI clinical trials recommendations: soluble biomarker assessments in clinical trials in osteoarthritis. Osteoarthritis Cartil 2015; 23: 686-697.

\section{*Correspondence to}

Weidong Zheng

Department of Traumatic Surgery

Jining Shandong First People's Hospital

PR China 